with a high domiciliary delivery rate, and I think that properly conducted domiciliary and maternity home care has its place. We do not want to lose it because the statistics are being misinterpreted or because of failures in other areas.-I am, etc.,

Ipswich, Suffolk.

ROY WEBB.

draw the attention of those obstetricians who carry out foetal samples to the two points made above-namely, to insert the blade at right angles to the scalp surface, and to examine the plastic blade-holder after completing the manouvre.-We are etc.,

\section{Broken Blade in Foetal Blood Sampling}

SIR,-We write to describe a complication of foetal blood sampling which to our knowledge has not previously been described. In brief, this is the breaking of the blade of the sample knife and its retention in the foetal scalp. Two cases have occurred; these will be reported in full elsewhere.

The first case (under the care of H.R.M.R.) occurred on the night of 31 December 1968 ; the sample was being taken as meconium was present in the liquor. The cervix at this time was $7 \mathrm{~cm}$. dilated, fairly well applied to the head, which was not engaged and in the left occipito transverse position. The scalp skin was described as "fairly loose." A successful single stab was made. At this time the fact that the blade had broken flush with the end of the white plastic blade-holder was not recognized, and thus no action was taken. The fact that the blade was retained in the foetal scalp was only recognized when the baby was brought to the casualty department on 4 April 1969 "with something sticking out of its head." The 2 -mm. blade was removed by pulling the protruding edge with forceps.

Following this the knife has been inspected after each sampling procedure. On 5 May a second case (under the care of W. L.W.) occurred. The patient was being sampled on account of a rising foetal heart by a different resident to the one involved in the first case. Both residents had considerable previous experience in the technique of foetal blood sampling. The cervix was $5 \mathrm{~cm}$. dilated, well applied to the head, which was $1 \mathrm{~cm}$. above the ischial spines and lying occipito posteriorly. A medium amnioscope $(1.5 \mathrm{~cm}$. diameter) was being used. In this case the blade was found to have broken at the same point when the latter was inspected. Following delivery, radiological investigation showed the blade to be lying with its flat surface parallel to the right parietal bone. The blade was removed by incising over the stab wound.

These two cases illustrate a previously undescribed hazard of foetal blood sampling, and, as illustrated in the first case, one that may not have been recognized. Prevention, however, is much more important. Simple experiments have shown that the knife blade does not break if it enters the skin at right angles. A similar experiment, inserting the blade at an angle of $30^{\circ}-45^{\circ}$, with the amnioscope held obliquely to the surface of the scalp, with a final correction to $90^{\circ}$, left the blade in the position found in the second case. This situation was aggravated by stabs in lines of "latitude" on the scalp rather than "longitude."

These findings have been reported to the manufacturers. The two blades and second white plastic blade-holder have been sent to them for examination. We would, however,
H. R. M. ROBERTS.

W. L. WhitehousE.

Queen Mary's Hospital,
London S.W.15.

\section{Glasgow Medical Women}

SIR,-As one of the respondents to the questionary by Drs. Morag C. Timbury and Maria R. Ratzer (10 May, p. 372) there appeared to me one glaring omission in an otherwise excellent article. This was the absence of information on the attitudes of the husbands of the married women, who comprised three-quarters of the group. Was this implied by the 13 women who regarded medical work as unsuitable for married women or those who found difficulty combining career and domestic responsibilities ? A sufficiency of child-minding services, good tax allowances, etc., would be of no avail if the career-minded woman doctor has not a co-operative husband; and by co-operating does he miss out somewhere, if not in his career then emotionally ?

If the career-minded woman doctor provides a child-minding mother substitute, should she also arrange a wife substitute, or perhaps the husband might be in danger of doing that himself ?-I am, etc.,

\section{JEAN MCEWEN,} Part-time Medical Officer,

Dumbarton. Dunbarton County Council.

SIR,-I see from the survey of Glasgow Medical Women 1951-4 (10 May, p. 372) that I am one of the men whose wife Dr. Morag C. Timbury and her co-author consider to be unemployed.

I do not want to belittle married women doctors who receive financial reward for their contribution to medicine, but I think that it is a little disparaging to describe the others as unemployed. Apart from giving our three teenage children a happy and secure childmedical wives who give their services voluntarily to the cause of medicine.-I am, etc.,

Royal Infirmary,
Glasgow C.4.

Shedden Alexander.

Glasgow C.4.

\section{“Normal " Values}

SIR,-Dr. H. Muller (3 May, p. 315) has voiced the distress of the practising physician struggling to cope with the ever-increasing number of pathology tests and the problem of remembering norms. His suggestion of expressing all test results as a percentage of the normal introduces one simplification, but it is insufficient, as he himself recognizes when he says that the normal may have a greater or lesser range and that it will be hood background, my wife is one of many necessary to remember this.
I would like to suggest that this problem, too, can be solved by a method of recording all test results, which is simple and requires little effort. ${ }^{1}$ This method expresses all test results in "standard" units. It has been argued that standard units imply a " normal " distribution of test results, but this is not so. In any case, for the sake of ease and interpretation, any distribution could be normalized first.

In order to avoid the difficulties of positive and negative scores as well as fractional ones, the easiest way to express the standard scores is in the form of " $T$ " scores. In this system the mean is always 50 , and the standard deviation is 10 . No fractions need be used, since an accuracy to the tenth of a standard deviation is quite sufficient; what does it signify to the clinician? It would tell him that a score of 50 indicated the average (for the particular group of patients he is considering); that a score just above or below 50 is one which is very close to the average. That a score between 40-60 covers about two-thirds of the population and is quite normal. That a score between 30 and 70 covers $95 \%$ of the population, and, although a score near a little above or a little close to the ends of the range is unusual, it is within normal limits. A score between 20 and 30 or between 70 and 80 is undoubtedly suspect, and above 20 and below 80 is definitely abnormal. Once this has been learned, it applies to all clinical tests-past, present, and future-known tests and those yet to be described. For those interested in details there will always be conversion tables which would change back the " $T$ " score to the actual quantity measured.

The one difficulty with this method is not that of comprehension, for, although the notions of distribution and frequency are still not everyday occurrences in the medical world, their understanding is rapidly increasing and spreading. The real difficulty is the fact that most tests are introduced with insufficient preliminary work to establish the norms. It is deplorable that the practice of medicine involves the use of tests for which the norms are not adequately known and not adequately reproduced, and that decisions should be made upon laboratory results which are sometimes meaningless. It is to be hoped that an increasing understanding of statistics will end in the abolition of the worship of figures produced by laboratories. -I am, etc.,

Department of Psychiatry,

Max Hamilton.

\section{REFERENCE}

1 Hamilton, M., Lancet, 1958, 1, 977.

\section{Treatment of Angina Pectoris}

SIR,-The publication of both a leading article and a paper on the treatment of angina pectoris (19 April, pp. 134 and 155) prompts me to offer some observations on the methodology of clinical trials in this field.

I agree with Dr. D. F. Wilson and colleagues that individual dose titration is necessary in such studies, as there are considerable limitations to a fixed dose method. I also agree that quantitative data can be misleading owing to increased effort tolerance on an active drug, but $I$ have found no reliable way of overcoming this difficulty. With regard 